\title{
Acesso aos serviços de saúde bucal de adultos e idosos
}

\section{Camila Santa Rosa Nunes ${ }^{1}$, Marlucilena Pinheiro da Silva ${ }^{2}$ e Ana Rita Pinheiro Barcessat ${ }^{3}$}

\footnotetext{
1 Mestranda em Ciências da Saúde pela UNIFAP, Especialista em Endodontia pela UNIASSELVI e em Gestão em Saúde pela UNIFAP, graduada em Odontologia pela Universidade Federal do Pará. Cirurgiã-dentista da Universidade Federal do Amapá, Brasil. E-mail: camilasrnunes@gmail.com

2 Doutora em Educação pela Universidade Federal de Uberlândia, Mestre em Saúde Coletiva pela Universidade de Fortaleza e graduada em Enfermagem e Obstetrícia pela Escola de Enfermagem Magalhães Barata. Professora Adjunto da Universidade Federal do Amapá e Enfermeira da Unidade Básica de Saúde da UNIFAP. Docente da Residência Multiprofissional em Saúde Coletiva, do curso de Graduação em Enfermagem e do Mestrado em Ciências da Saúde da UNIFAP, Brasil. E-mail: marlucilena@unifap.br

3 Doutora em Ciências: Patologia Oral e Maxilofacial pela FO-USP, Mestre Profissional Laser em Odontologia pelo IPEN-FOUSP e Cirurgiã Dentista pela Universidade Federal do Pará. Professora Adjunto da Universidade Federal do Amapá, no curso de Graduação em Enfermagem e no Programa de Pós-Graduação em Ciências da Saúde (Mestrado), Brasil. E-mail: ritabarcessat@gmail.com
}

RESUMO: O acesso aos serviços de saúde bucal é um campo de estudo relevante, pois permite inferir dados quanto à utilização e necessidade dos serviços, além de auxiliar no planejamento e na organização das ações de saúde. Trata-se de uma revisão integrativa cujo objetivo é sintetizar a produção científica brasileira acerca da acessibilidade dos usuários adultos e idosos aos serviços de saúde oral. A seleção foi baseada entre cruzamentos dos descritores: acesso aos serviços de saúde, saúde bucal e assistência odontológica, na base de dados Biblioteca Virtual em Saúde. Foram selecionados 20 artigos divididos por similaridade temática: acesso aos serviços de saúde geral, acesso aos serviços de saúde bucal de adultos, acesso aos serviços de saúde bucal de idosos. A partir deste levantamento identificou-se como fatores relacionados ao maior uso destes serviços: ser do sexo feminino, ter maior renda e escolaridade. A dor e a maior percepção de saúde são os maiores motivos para a procura de atendimento. A efetivação de um acesso equitativo é um desafio para o Sistema de Saúde e o desenvolvimento de estudos que busquem conhecimento desses fatores é de suma importância para a redução dessas desigualdades.

Palavras chave: Saúde bucal. Acesso aos serviços de saúde. Assistência odontológica. Assistência odontológica para idosos. Qualidade, acesso e avaliação da assistência à saúde.

\section{Adult and elderly access to oral health services}

ABSTRACT: Access to oral health services is a relevant field of study, as it allows to infer data on the use and need of services, and assist in the planning and organization of health actions. This integrative review aimed to synthesize the Brazilian scientific production about the accessibility of adult and elder users to oral health services. The selection was based interweaving of descriptors: access to health services, oral health and dental care, in the database of Health Virtual Library. Twenty articles were selected divided by thematic similarity: access to general health services, adult access to dental services, access to oral health services for the elderly. From this survey it was identified as factors associated with increased use of these services: being female, have higher income and education. The pain and the greater perception of health are the main reasons for seeking care. The enforcement of equitable access is a challenge for the Health System and the development of studies that seek knowledge of these factors is very important to reduce these inequalities.

Keywords: Oral health. Health services accessibility. Dental care. Dental care for aged. Health care quality, access, and evaluation. 


\section{INTRODUÇÃO}

O artigo 196 da Constituição Federal (1988) possibilitou profundas mudanças no que se refere ao nosso sistema de saúde. Tal artigo afirma que a saúde é direito de todos e dever do Estado, garantido mediante políticas sociais e econômicas que visem à redução do risco de doença e de outros agravos e ao acesso universal e igualitário às ações e serviços para sua promoção, proteção e recuperação (BRASIL, 1988).

Dentro deste contexto, a saúde bucal constitui-se num elemento de grande relevância, pois a mesma apresenta reflexos na saúde geral e qualidade de vida da população. Porém, tal importância só veio a ser reconhecida nos últimos anos, principalmente com o avanço da Política Nacional de Saúde Bucal- Brasil Sorridente (2004), que constitui num conjunto de ações nos âmbitos individual e coletivo que abrange a promoção da saúde, a prevenção de agravos, o diagnóstico, o tratamento e a reabilitação, com ampliação do acesso ao tratamento odontológico gratuito aos brasileiros por meio do Sistema Único de Saúde (SUS) (COSTA; CHAGAS; SILVESTRE, 2006).

As principais linhas de ação do Programa Brasil Sorridente são a reorganização da Atenção Básica em saúde bucal com a implantação das Equipes de Saúde Bucal na Estratégia Saúde da Família; a ampliação e qualificação da Atenção Especializada, em especial com a implantação de Centros de Especialidades Odontológicas e Laboratórios Regionais de Próteses Dentárias além da viabilização da adição de flúor nas estações de tratamento de águas de abastecimento público (MACHADO, 2012).

Apesar da implementação de tais políticas, e da garantia ao direito à saúde firmada pela Constituição Federal, o acesso a esses serviços nem sempre são respeitados, se tornando muitas vezes seletivo, focalizado e excludente, mesmo este sendo um atributo essencial para o alcance da qualidade nos serviços de saúde (ASSIS; JESUS, 2012).

A procura por serviços odontológicos está na terceira posição entre os motivos de busca por serviço de saúde, sua proporção aumentou de $8,5 \%$ para $14,0 \%$ no período de 2003 a 2008, o que equivale, em termos absolutos, ao acréscimo de aproximadamente 10 milhões de pessoas (PERES et al., 2012). O acesso a esses serviços é de extrema importância, pois é o primeiro contato que o usuário tem com os serviços de saúde.

Para Andersen (1995), a relação direta entre acesso e utilização de serviços de saúde é dividida em acesso potencial que consiste na procura e no contato inicial ao serviço; o acesso realizado que se resume na utilização do serviço; o acesso efetivo que se relaciona ao processo de cuidado durante o período de utilização do serviço; e $o$ acesso eficiente percebido pelas alterações nas condições de saúde e no contentamento das pessoas levando-se em consideração os serviços e a qualidade dos mesmos disponibilizados durante o seu percurso (ANDERSEN, 1995).

Segundo o modelo comportamental de Andersen e Newman (2005), o acesso aos serviços de saúde é influenciado por fatores predisponentes, que existem antes mesmo da ocorrência do problema. Temos como exemplo: gênero, idade, entre outros. Além da influência por fatores capacitantes definidos como meios que possibilitam ou impedem o uso dos serviços, e pelas necessidades de saúde (condições de saúde percebidas pelas pessoas - auto percepção) ou 
diagnosticadas por profissionais de saúde (ANDERSEN; NEWMAN, 2005).

A acessibilidade aos serviços de saúde é um campo de estudo relevante, pois permite inferir dados quanto à utilização e necessidade dos serviços, bem como auxiliar no planejamento e organização das ações de saúde (PINTO; MATOS; LOYOLA FILHO, 2012). Portanto, o objetivo desta revisão integrativa de literatura é identificar a produção científica acerca da acessibilidade dos usuários aos serviços de saúde bucal no Brasil, no período de 2009 a 2016.

\section{METODOLOGIA}

Trata-se de uma revisão integrativa com base em produções científicas selecionadas na base de dados da Biblioteca Virtual em Saúde (BVS) a respeito do acesso aos serviços de saúde bucal da população brasileira adulta e idosa. Tal base de dados foi selecionada, pois possibilita uma busca simultânea das publicações mais relevantes efetuadas em campo nacional. A questão norteadora deste estudo foi: "Qual o grau de acessibilidade da população brasileira adulta e idosa aos serviços de saúde bucal?".

Para a realização da pesquisa foram selecionados os seguintes termos indexados nos Descritores em Ciências da Saúde (DeCS): acesso aos serviços de saúde, saúde bucal e assistência odontológica, sendo realizadas todas as possibilidades de cruzamento, utilizando os ícones AND e OR. Optou-se por periódicos publicados no período de janeiro de 2009 a dezembro de 2016. As pesquisas restringiram-se ao ambiente nacional, sendo excluídos publicações e estudos efetuados em outros países. Outro critério de seleção foi seu resumo apresentar informações quanto à acessibilidade aos serviços de saúde geral e de saúde bucal, bem como a apresentação de indicadores de tais serviços. Após esta etapa foram selecionados 64 artigos, os quais foram lidos na íntegra, observando se os mesmo correspondiam ao objetivo do trabalho. Em seguida foi realizada uma nova seleção, na qual restaram apenas 20 artigos, que foram divididos de acordo com as seguintes temáticas: acesso aos serviços de saúde geral, acesso aos serviços bucal de adultos, acesso aos serviços de saúde bucal de idosos.

\section{RESULTADOS E DISCUSSÃO}

\subsection{Acesso aos serviços de saúde geral}

O acesso universal aos serviços de saúde, além de ser uma garantia constitucional, é uma bandeira de luta dos movimentos sociais que buscam por reais possibilidades de mudança e melhora no atendimento aos problemas de saúde da população (JESUS; ASSIS, 2010).

A acessibilidade é um tema multifacetado e multidimensional que envolve aspectos políticos, econômicos, sociais, organizativos, técnicos e simbólico, no estabelecimento de caminhos para a universalização da sua atenção (ASSIS; JESUS, 2012; VIANA; MARTELLI; PIMENTEL, 2012).

A qualidade e ampliação do acesso dependem de diversos fatores que perpassam principalmente pela capacidade que o profissional desenvolve em avançar nesse processo e de capacidade de visualizar entraves e caminhos efetivos a serem construídos e percorridos, além da impotência apresentada por alguns gestores para gerar mudanças e da falta de participação da sociedade e dos trabalhadores na gestão que só fortalecem a supremacia dos interesses 
do mercado e contribuem para a garantia de um acesso limitado (BARBOSA; ELIZEU; PENNA, 2013; SILVA; BENITO, 2013).

A escolha desses profissionais deve ser criteriosa para que haja a superação de certos movimentos do cotidiano de trabalho, como tomada de decisão, capacidade de governança dessas equipes e gestão do cuidado, que ultrapassem o modelo curativista hegemônico para então possibilitar a ampliação do acesso e maior resolutividade dos casos. Fornecer mecanismos apropriados que facilitem o alcance aos diferentes níveis de atenção à saúde, como acolhimento e práticas mais humanizadas, são tecnologias necessárias para a resolutividade dos casos (BARBOSA; ELIZEU; PENNA, 2013). Para tal, as políticas de saúde devem ser capazes de eliminar a barreira financeira entre os serviços e a comunidade, de enfrentar a mercantilização do setor saúde e a desproporção oferta/demanda existente; e construindo uma rede de atenção regionalizada e hierarquizada que garanta o acesso universal, equitativo e integral (ASSIS; JESUS, 2012).

\subsection{Acesso aos serviços de saúde bucal de adultos}

Os serviços públicos de atenção à saúde bucal, no Brasil, historicamente caracterizaram-se pelo privilegiamento de um determinado segmento populacional, que são crianças e adolescentes em idade escolar, em detrimento da atenção a outros segmentos como os adultos, além de focar muitas vezes seu atendimento na demanda espontânea (PINTO; MATOS; LOYOLA FILHO, 2012; PERES, 2012, VIANA; MARTELLI; PIMENTEL, 2012).

Inquéritos Epidemiológicos, como o Saú- de Bucal Brasil (SB Brasil) e a Pesquisa Nacional por Amostras de Domicílio (PNAD) relativos à saúde bucal têm apontado um quadro preocupante para os adultos jovens, onde tem se constatado, uma elevada proporção de dentes perdidos, além da maior necessidade de tratamento protético e outros procedimentos especializados (FUNDAÇÃO INSTITUTO BRASILEIRO DE GEOGRAFIA E ESTATÍSTICA, 1998; PERES, 2012; SOUZA, 2012).

A utilização desses serviços pela população brasileira é baixa, e reduz com o avançar da idade. Em 2003, os adultos brasileiros apresentavam índice CPO-D médio (índice de saúde bucal que traduz a experiência de cárie do indivíduo ao longo da vida) superior a 20 dentes, sendo o componente que representa os dentes perdidos (sem possibilidade de recuperação) superior a $60 \%$. Além de um baixo percentual populacional (próximo a 22\%) com periodonto saudável (sem alterações como sangramento gengival, cálculo ou bolsa periodontal) (PINTO; MATOS; LOYOLA FILHO, 2012). A baixa utilização dos serviços bucais pode ser explicada por fatores como as barreiras de acesso enfrentadas pela população e a disponibilidade desses serviços, bem como as características dos sistemas municipais de saúde (SOUZA, 2012).

A distribuição das redes de saúde e a oferta de serviços são profundamente desiguais entre as regiões. Há uma maior utilização de serviços públicos nas regiões Nordeste e Sul, comparativamente à região Sudeste, e em municípios de pequeno porte, comparativamente aos de maior porte. 0 maior uso dos serviços públicos pelos municípios de pequeno porte pode ser justificado pelo menor poder aquisitivo de sua população e pela menor disponibilidade de 
serviços odontológicos privados (PINTO; MATOS; LOYOLA FILHO, 2012; VIANA; MARTELLI; PIMENTEL, 2012).

No último levantamento do SBBrasil 2010, a região Norte apresentou a maior proporção de indivíduos que nunca foram ao dentista, maior intervalo de tempo desde a última consulta e a maior proporção de consultas motivadas pela dor do que no Sul e Sudeste. Já para as regiões Sul e Sudeste, juntamente com as capitais, foi observada uma maior taxa de uso de serviços particulares, o que demonstra que o SUS ainda não consegue atender plenamente a demanda por atendimento odontológico da população (MACHADO, 2012; PERES, 2012).

A Agência Nacional de Saúde Suplemen$\operatorname{tar}$ (ANS) revelou em 2010 que os planos de assistência médica cobrem $23 \%$ da população no Brasil, com maior cobertura nos Estados das regiões Sudeste e Sul, nas capitais e nas regiões metropolitanas. Os planos exclusivamente odontológicos atingem apenas $7,1 \%$ da população brasileira (PERES, 2012).

A população com maior renda e maior escolaridade apresenta maior prevalência de uso regular dos serviços odontológicos, sendo que a maior renda pode facilitar a compra de um serviço e a escolaridade pode trazer a informação da importância do uso regular de serviços de saúde odontológico e aumenta a auto percepção de saúde bucal (PINTO; MATOS; LOYOLA FILHO, 2012; MACHADO, 2012; MIRANDA; PERES, 2013; SOUZA; CHAVES, 2011; SOARES; CHAVES; CANGUSSU, 2013).

A população menos favorecida socioeconomicamente é a que mais utiliza os serviços públicos odontológicos, devido à impossibilidade de custeio dos serviços privados (PINTO; MATOS; LOYOLA FILHO, 2012;
SOUZA; CHAVES, 2011; SOARES; CHAVES; CANGUSSU, 2013). Estas desigualdades entre os grupos sociais ainda é grande e podem ser consideradas ética e politicamente inaceitáveis, uma vez que revelam desvantagens de acesso e de utilização dos serviços odontológicos entre os mais pobres, indo de encontro a um princípio do Sistema Único de Saúde, o da equidade (PERES et al., 2012; PINTO; MATOS; LOYOLA FILHO, 2012).

Os usuários que se autodeclaram pardos apresentam uma taxa de $35 \%$ maior de falta de acesso a serviços odontológicos do que os indivíduos brancos, este dado pode estar relacionado ao fato da população negra (pardos + pretos), que é em sua maioria mais desfavorecida socioeconomicamente, apresentar maior prevalência e maior complexidade de agravos bucais, resultando em necessidades de tratamento mais complexas e não atendidas pelo SUS, majoritariamente utilizado por este grupo populacional (PERES, 2012).

A dor ainda é uma das maiores motivações para a procura do atendimento odontológico, o que reflete a não utilização rotineira de serviços odontológicos, sendo na maioria das vezes realizada apenas a consulta de urgência, que demandará apenas um atendimento curativo. Tal atendimento apenas reforça o atual quadro de serviços públicos que é reconhecido apenas por realizar extrações e efetuar procedimentos curativos, em detrimento da prevenção e proservação (CHAVES, 2012; ESTEVES, 2012; PINTO; MATOS; LOYOLA FILHO, 2012; MACHADO, 2012). O uso regular dos serviços de saúde bucal é característico apenas dos usuários que usufruem do setor privado (PINTO; MATOS; LOYOLA FILHO, 2012; MACHADO, 2012). 
As mulheres são as que mais utilizam, de forma regular ou não, os serviços públicos odontológicos, isto pode ser justificado pela maior disponibilidade de tempo para comparecer às unidades de saúde pública no horário de funcionamento, em razão das mulheres apresentarem um nível de ocupação (percentual de pessoas ocupadas) mais baixo que os homens, uma maior preocupação e autocuidado com a saúde (CASTRO; OLIVEIRA; ARAÚJO, 2011; CHAVES, 2012; PINTO; MATOS; LOYOLA FILHO, 2012; MACHADO, 2012; MIRANDA; PERES, 2013; SOUZA; CHAVES, 2011).

A percepção da necessidade de tratamento por parte dos usuários é um fator que amplia a busca pelo atendimento odontológico, apresentando-se como importante indicador que pode determinar a utilização dos serviços de forma regular, podendo ser influenciada por medidas de educação em saúde (CASTRO; OLIVEIRA; ARAÚJO, 2011; MACHADO, 2012).

O serviço público nem sempre é bem avaliado pelos usuários, sendo que essa insatisfação pode derivar do tempo de espera pelo atendimento, da impossibilidade de escolha do profissional, do não atendimento de todas as necessidades e da desorganização dos processos de trabalho dos profissionais (PINTO; MATOS; LOYOLA FILHO, 2012).

A situação de grande necessidade acumulada reflete o histórico descaso desse segmento populacional na atenção à saúde bucal em nosso país e coloca um grande desafio ao SUS, considerando o seu papel na redução das desigualdades e de provimento de acesso universal ao cuidado integral (PINTO; MATOS; LOYOLA FILHO, 2012).

\subsection{Acesso aos serviços de saúde bucal dos idosos}

O fenômeno da transição demográfica gera para o Brasil um crescente aumento no número de idosos que tem ocorrido de forma rápida e intensa. Tal mudança nem sempre vem acompanhada de modificações no atendimento às necessidades de saúde desse grupo populacional, apesar de as mesmas estarem garantidas por lei. A Lei Orgânica da Saúde, bem como a Política Nacional do Idoso (Lei no 8842/1994), que possui como objetivo a promoção do enveIhecimento saudável, a manutenção e a meIhoria da capacidade funcional dos idosos, a prevenção de doenças, a recuperação e a reabilitação da saúde e a criação do Estatuto do Idoso em 2003 (Lei no 10741), que garantiu ao idoso a assistência à saúde, nos diversos níveis de atendimento do SUS, através de programas e medidas profiláticas, foram medidas adotadas na tentativa de atender melhor as necessidades dessa população (FERREIRA; ANTUNES; ANDRADE, 2013; KOCH FILHO, 2011; VIANA, 2010).

Apesar da ampliação da oferta e da cobertura da assistência odontológica no Brasil, a utilização do serviço por idosos é ainda muito inferior às outras faixas etárias, diante de uma demanda acumulada de tratamento e a alta prevalência de perda dentária desacompanhada de reabilitação protética, resultado de uma história de modelo de saúde bucal individualista de baixa complexidade e pela ausência de programas específicos para esse grupo. No projeto Saúde Bucal Brasil, realizado em 2003 e 2010, foi observado um aumento no número de idosos que nunca frequentaram o dentista, esse índice que era de 5,8\% passou a ser de 14,7\% (FERREIRA; ANTUNES; ANDRADE 
2013).

Dentre os fatores limitadores ao acesso dessa população aos serviços de saúde bucal, podemos citar a: acessibilidade geográfica, caracterizada pelo adequado planejamento da localização dos serviços de saúde, entre outros fatores, como a distância, o tempo necessário para cobri-la e os meios de transporte aceitáveis para a população; a acessibilidade econômica, que seria a remoção de barreiras derivadas do sistema de pagamento ou contribuição pelo usuário; a acessibilidade cultural, adequação das normas e técnicas aos hábitos e costumes da população usuária; e a acessibilidade funcional, ofertas de serviços oportunos e adequados à necessidade da população (FERREIRA; ANTUNES; ANDRADE, 2013; $\mathrm{KOCH}$ FILHO, 2011; VIANA, 2010). Diferentes níveis econômicos, culturais e de saúde também interferem no acesso, na aceitação, na realização e no sucesso dos tratamentos odontológicos (KOCH FILHO, 2011).

Koch (2011) considera que os principais motivos que impedem os idosos de procurar os serviços odontológicos são: a falta de percepção de necessidade, os altos custos do tratamento odontológico, o comprometimento motor ou mental, experiências negativas anteriores, a falta de informação sobre os cuidados com a boca e os tabus pessoais como a perda de dente com o passar da idade é algo normal. (FERREIRA; ANTUNES; ANDRADE, 2013; KOCH FILHO, 2011; VIANA, 2010). Simões e Carvalho (2011), consideram como principais barreiras de acesso aos serviços odontológicos a baixa escolaridade, a baixa renda e a escassa oferta de serviços públicos de atenção à saúde bucal.

Idosos com maior renda e nível de escolaridade apresentaram maiores prevalên- cias de uso recente e regular do serviço odontológico. A maior renda facilitará a compra de um serviço ou facilitará o acesso ao mesmo, e a maior escolaridade possibilitará um maior conhecimento a respeito da importância do uso regular de serviços de odontologia e da saúde bucal (PERES et al, 2012; FERREIRA; ANTUNES; ANDRADE, 2013; VIANA, 2010).

A falta de percepção da necessidade de atendimento odontológico aliado à cultura de que indivíduos edêntulos não necessitam de acompanhamento odontológico contribuem para o número reduzido de idosos frequentadores dos serviços de saúde bucal, refletindo a falta de orientação e de cuidados com a saúde bucal.

Quando se realiza a análise entre a utilização dos serviços odontológicos e a variável raça, observa-se que o mesmo é um fator limitante na utilização dos serviços odontológicos, sendo que a chance de um idoso negro nunca ter consultado um dentista é duas vezes maior quando comparado a um idoso branco (SOUZA, 2012).

O reduzido número de programas de saúde bucal voltados a 3a idade e o baixo índice de profissionais e com pouco preparo para o atendimento desses indivíduos em nosso país, realçam a necessidade de profundos estudos e adequação técnica para atuação nessa população. A saúde bucal do idoso brasileiro encontra-se em situação precária, existindo ainda uma demanda reprimida no sentido de ofertas de recursos e serviço, além do elevado índice de edentulismo, o que reflete a ineficácia historicamente presente nos serviços públicos de atenção odontológica, que não foca na prevenção e promoção de saúde para esse grupo populacional, sendo muitas vezes apenas curativista (SIMÕES; CARVALHO, 
2011; VIANA, 2010).

\section{CONCLUSÃO}

O acesso aos serviços odontológicos de adultos e idosos ainda é muito ineficiente, se tornando um grande desafio ao Sistema Único de Saúde. Para tanto, se faz necessário que a gestão pública intensifique os esforços e recursos no sentido de aumentar a cobertura, a resolutividade e a qualidade das ações, para que possa, efetivamente, cumprir o seu papel na produção da saúde bucal, até mesmo buscando desenvolver programas específicos que possam assegurar serviços odontológicos às classes desassistidas. É responsabilidade das esferas governamentais quebrar as barreiras que dificultam esse acesso, bem como contribuir para diminuir as disparidades de utilização desse serviço entre as diferentes regiões do Brasil, buscando fortalecer o trabalho preventivo em contrapartida ao modelo curativista. Tais medidas resultarão num maior acesso da população a esse serviço bem como diminuirá diferenças intrínsecas encontradas nos mais diversos grupos populacionais.

O momento atual é oportuno para aquisição e consolidação de novas práticas, pois há de se considerar que em se tratando de saúde bucal, um grande passo foi dado através da implantação da atual Política Nacional destinada ao setor, porém, é necessário que haja, além de incentivos para implantação de novas políticas públicas de saúde bucal, um maior suporte e capacitação técnica para os trabalhadores da saúde, traduzindo-se em mudanças efetivas no modo de produção e organização do sistema de saúde.

\section{REFERÊNCIAS}

ANDERSEN, R. M. Revisiting the behavioral model and access to medical care: does it matter? Journal of health and social behavior, p. 1-10, 1995.

ANDERSEN, R.; NEWMAN, J. F. Societal and individual determinants of medical care utilization in the United States. The Milbank Quarterly, v. 83, n. 4, 2005.

ASSIS, M. M. A.; JESUS, W. L. A. Acesso aos serviços de saúde: abordagens, conceitos, políticas e modelo de análise. Ciência \& Saúde Coletiva, v. 17, n. 11, 2012.

BARBOSA, S.; ELIZEU, T. S.; PENNA, C. M. M. Ótica dos profissionais de saúde sobre o acesso à atenção primária à saúde. Revista Ciência \& Saúde Coletiva, v. 18, n. 8, 2013. BRASIL. Constituição (1988). Constituição da República Federativa do Brasil. Brasília, DF, Senado Federal, 1988.

CASTRO, R. D.; OLIVEIRA, A. G. R. da C.; ARAÚJO, I. M. Estudo da Acessibilidade Organizacional aos Serviços de Saúde Bucal de um Município de Pequeno Porte do Nordeste Brasileiro. Revista Brasileira de Ciências da Saúde, v. 14, n. 4, p. 95-76, 2011.

CHAVES, S. C. L. et al. Características do acesso e utilização de serviços odontológicos em municípios de médio porte. Ciência \& Saúde Coletiva, v. 17, n. 11, 2012.

COSTA, J. F. R.; CHAGAS, L. de D.; SILVESTRE, R. M. A política nacional de saúde bucal do Brasil: registro de uma conquista histórica. In: Técnica. Desenvolvimento de Sistemas e Serviços de Saúde. OPAS, 2006. ESTEVES, R. S. de S. Perfil da atenção odontológica de um município de grande porte do Brasil. 2012. 118 f. Tese (Doutorado) Curso de Odontologia, Faculdade de Odontologia, Universidade Federal de Minas Gerais, Belo Horizonte, 2012. 
FERREIRA, C. de O.; ANTUNES, J. L. F.; ANDRADE, F. B. Fatores associados à utilização dos serviços odontológicos por idosos brasileiros. Revista de Saúde Pública, v. 47, n.3, p. 90-97, 2013.

FUNDAÇÃO INSTITUTO BRASILEIRO DE GEOgRAFIA E ESTATÍSTICA. Pesquisa Nacional por Amostra de Domicílios (PNAD 98). Rio de Janeiro, 1998.

JESUS, W. L. A.; ASSIS, M. M. A. Revisão sistemática sobre o conceito de acesso nos serviços de saúde: contribuições do planejamento. Ciência \& Saúde Coletiva, v. 15, n. 1, 2010.

KOCH FILHO, H. R. et al. Uma década da Odontogeriatria brasileira. Archives of Oral Research, v. 7, n. 3, 2017.

MACHADO, L. P. et al. Uso regular de serviços odontológicos entre adultos e idosos em região vulnerável no sul do Brasil. Revista de Saúde Pública, v. 46, n. 3, p. 526-533, 2012.

MIRANDA, C. D. C.; PERES, M. A. Determinantes da utilização de serviços odontológicos entre adultos: um estudo de base populacional em Florianópolis, Santa Catarina, Brasil. Cadernos de Saúde Pública, v. 29, n. 11, p. 2319-2332, 2013.

PERES, K. G. et al. Redução das desigualdades sociais na utilização de serviços odontológicos no Brasil entre 1998 e 2008. Revista de Saúde Pública, v. 46 , n. 2, p. 250-258, 2012.

PERES, M. A. et al. Desigualdades no acesso e na utilização de serviços odontológicos no Brasil: análise do Sistema de Vigilância de Fatores de Risco e Proteção para Doenças Crônicas por Inquérito Telefônico (VIGITEL 2009). Caderno de Saúde Pública, v. 28, 2012.

PINTO, R. da S.; MATOS, D. L.; LOYOLA FILHO, A. I. Características associadas ao uso de serviços odontológicos públicos pela população adulta brasileira. Ciência \& Saúde Coletiva, v. 17, n. 2, 2012.

SILVA, B. F. S.; BENITO, G. A. V. A voz de gestores municipais sobre o acesso à saúde nas práticas de gestão. Ciência \& Saúde Coletiva, v. 18, n. 8, p. 2189-2200, 2013.

SIMÕES, A. C. de A.; CARVALHO, D. M. The reality of the oral/dental health of the elderly in the Brazilian Southeast. Ciência \& Saúde Coletiva, v. 16, n. 6, p. 2975-2982, 2011.

SOARES, F. F.; CHAVES, S. C. L.; CANGUSSU, M. C. T. Desigualdade na utilização de serviços de saúde bucal na atenção básica e fatores associados em dois municípios brasileiros. Revista Panamericana de Salud pública, v. 34, n. 6, p. 401-406, 2013.

SOUZA, E. H. A. et al. Raça e o uso dos serviços de saúde bucal por idosos. Ciência \& Saúde Coletiva, v. 17, n. 8, 2012.

SOUZA, L. F.; CHAVES, S. C. L.. Política Nacional de Saúde Bucal: acessibilidade e utilização de serviços odontológicos especializados em um município de médio porte na Bahia. Revista Baiana Saúde Pública, v. 34, n. 2, 2011.

VIANA, A. A. de F. et al. Acessibilidade dos idosos brasileiros aos serviços odontológicos. RFO UPF, v. 15, n. 3, p. 317-322, 2010. VIANA, I. B.; MARTELLI, P. J. de L.; PIMENTEL, F. C. Análise do acesso aos serviços odontológicos através do indicador de primeira consulta odontológica programática em Pernambuco: estudo comparativo entre os anos 2001 e 2009. Revista Brasileira em Promoção da Saúde, v. 25, n. 2, p. 151-160, 2012.

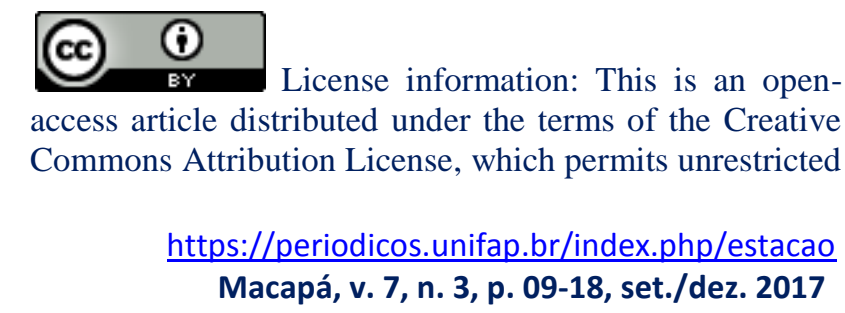


use, distribution, and reproduction in any medium, provided the original work is properly cited.

Artigo recebido em 14 de agosto de 2017.

Avaliado em 17 de novembro de 2017.

Aceito em 12 de dezembro de 2017.

Publicado em 19 de dezembro de 2017.

\section{Como citar este artigo (ABNT):}

NUNES, Camila Santa Rosa; SILVA, Marlucilena Pinheiro da; BARCESSAT, Ana Rita Pinheiro. Acesso aos serviços de saúde bucal de adultos e idosos. POTTMEIER. Blumenau. Estação Científica (UNIFAP), Macapá, v. 7, n. 3, p. 09-18, set./dez. 2017. 